\title{
Survey Paper on PEGASIS: Energy Conservation in Heterogeneous Environment
}

\author{
Bhatt Bhargavi, Prof. Chandresh R. Parekh \\ Department of Wireless and Mobile Computing GTU PG-School, BISAG Gandhinagar, India \\ Department Of Electroctonics and Communication Governmnt Engineering College, Gandhinagar, India.
}

\begin{abstract}
Wireless sensor network is vast area for research. Because of scarce resources, energy consumption and battery lifetime is crucial aspects in WSN. So with the help of routing protocol, improvement can be done. More energy can be conserved and network lifetime can be improved by modifying the existing protocols. Power Efficient GAthering in Sensor Information System (PEGASIS) is an improvement of hierarchical protocol. It mainly focuses on network lifetime. It uses chaining construction and data gathering approach to communicate. Aggregated data will pass on to the head node and it will pass the data to the base station. This paper introduces the PEGASIS, discusses the related previous work. New aspect of PEGASIS in heterogeneous environment is proposed. The new scheme is proposed in order to conserve energy.
\end{abstract}

Keywords: Wireless Sensor Network, PEGASIS, Energy.

\section{INTRODUCTION}

The origin of WSNs can be seen in military and heavy industrial area. The first wireless sensor network was the Sound Surveillance System (SOSUS), developed by the United States Military in the 1950s to detect and track Soviet submarines. This network used submerged acoustic sensors - hydrophones - distributed in the Atlantic and Pacific oceans. This sensing technology is still in service today. Other applications of WSN are like undersea wildlife and volcanic activity.

The heart of any WSN lies in the sensors. The past decade has seen advancement in multiple sensing technologies:

- Micro electro mechanical systems (MEMS)

- CMOS-based sensors

- LED sensors ${ }^{[1]}$

What is WSN :

- $\quad$ Provide a bridge between the real physical and virtual worlds.

- $\quad$ Allow the ability to observe the unobservable at a fine resolution over large scales

- Have a wide range of potential applications

A. Topologies

There are mainly four types of network topologies:

- $\quad$ One-way communication

- $\quad$ Bi-directional

- $\quad$ Star topology

- $\quad$ Mesh topology

The first networking protocols were simple one-way communication, for example applications such as tire pressure monitoring systems and television remote controls. As per requirement advancement in topologies came into picture, networking engineers developed low-memory protocols for bi-directional, star and finally mesh technologies. In addition, the industry is making the transition from proprietary to standardized protocols, networking protocols such as Zig-Bee and its variants removes the burden of continuous development costs and frees.

B. Challenges

- $\quad$ Node deployment

- $\quad$ Energy Consumption with accuracy

- $\quad$ Node Heterogeneity

- $\quad$ Scalability

- $\quad$ Network Dynamics

- Connectivity

- $\quad$ Coverage

- Data Aggregation

- Quality of Service 


\section{PEGASIS}

The PEGASIS is hierarchical protocol. It is a near optimal chain-based protocol. The basic idea of the protocol is that, in order to extend network lifetime, nodes need only to communicate with their closest neighbors and they take turns in communicating with the BS. To increase network lifetime basic radio model is used. Transmit to and receive from formula of radio model are respectively as follows:

Where,

$$
\mathrm{E}_{\mathrm{TX}}(\mathrm{k}, \mathrm{d})=\mathrm{E}_{\text {elec }} * \mathrm{k}+\mathrm{Eamp}^{*} \mathrm{k}^{*} \mathrm{~d}^{2}
$$

$\mathrm{k}$ is a bit message at distance $\mathrm{d}^{[9]}$

Where,

$$
\mathrm{E}_{\mathrm{RX}}(\mathrm{k})=\mathrm{E}_{\text {elec }} * \mathrm{k}
$$

$\mathrm{E}_{\mathrm{TX} \text {-elec }}=$ Transmitter Electronic

$\mathrm{E}_{\mathrm{RX} \text {-elec }}=$ Receiver Electrionic

$\mathrm{E}_{\mathrm{TX} \text {-elec }}=\mathrm{E}_{\mathrm{RX} \text {-elec }}=\mathrm{E}_{\text {elec }}$

$\mathrm{E}_{\mathrm{TX}-\mathrm{elec}}=50 \mathrm{~nJ} / \mathrm{bit}$

PEGASIS has two main objectives:

1. Increase the lifetime of each node, and thereby increase the network lifetime.

2. Allow only local coordination between nodes those are close to each other, so that the bandwidth consumed in communication is reduced.

\section{Working of PEGASIS}

Basic PEGASIS uses one node in a chain to transmit to the BS and avoid duplication transmission. To get the information about closest neighbor node in PEGASIS, each node uses the signal strength to measure the distance to all neighboring nodes. After adjustment of the signal strength only one node can be heard. The chain in PEGASIS will consist of those nodes which are neighbor node from path to the base-station. For example following figure shows node 0 connecting to node 3 , node 3 connecting to node 1 , and node 1 connecting to node 2 in that order. When a node dies, the chain is reconstructed in the same manner to bypass the dead node. When the round of all nodes communicating with the BS ends, a new round will start and so on. This reduces the power required to transmit data per round as the power draining is spread uniformly over all nodes.

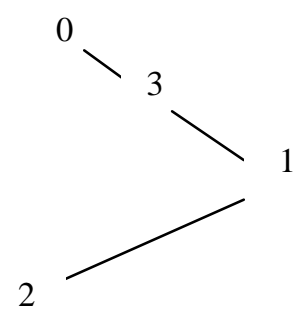

Figure1. Chain construction ${ }^{[6]}$

PEGASIS is able to increase the lifetime of the network twice as much the lifetime of the network under any other hierarchical protocol. The clustering overhead is avoided, PEGASIS still requires dynamic topology adjustment since a sensor node needs to know about energy status of its neighbors in order to know where to route its data. Such topology adjustment can introduce significant overhead especially for highly utilized networks. Moreover, PEGASIS assumes that each sensor node can be able to communicate with the BS. Sensor nodes use multi-hop communication to reach the base station. It also assumes that all nodes maintain a complete database about the location of all other nodes in the network. PEGASIS assumes:

- all sensor nodes have the same level of energy and they are likely to die at the same time.

- sensors will be fixed or immobile, some sensors may be allowed to move and hence affect the protocol functionality.

\section{RELATED WORK}

A. Performance Analysis of Concentric Cluster based PEGASIS for Wireless Sensor Networks

PEGASIS is essential to develop effective and energy efficient protocols in order to improve network lifetime. And one of the well-known chain-based routing protocols for improving energy efficiency. But this causes redundant data transmission since one of the nodes on the chain is selected as a head node. This problem is overcome by enhanced PEGASIS based on concentric clustering scheme. In concentric clustering scheme, network is divided in the form of concentric shaped clusters which has four steps, which are, 


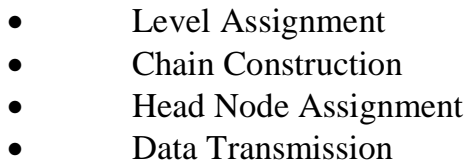

In this energy consumption is low compare to previous protocol. This is formulated,

$$
E=n * E_{\text {elec }} * k * E_{a m p} * \sum_{m=1}^{n}\left[d^{2}{ }_{(m-1, m)}\right]
$$

$\mathrm{E}_{\text {elec }}=$ per bit energy consumption in the transmitter circuitry

$\mathrm{K}=$ Data bits

$\mathrm{E}_{\mathrm{amp}}=$ Amplifier transmiotting energy

$\mathrm{d}_{\mathrm{ij}}=$ Distance from I node to $\mathrm{j}$ node

PEGASIS linear chain based scheme, as transmission is sequential average delay per round is still high thus simultaneous transmission is desired. If the nodes are CDMA capable, then binary scheme can be used to perform parallel communication to reduce overall delay. In Chain-based Binary Approach using CDMA, pair of data is done to delay possible delay. At the lowest level, chain is constructed in the same manner as was done in the PEGASIS. For data gathering round each node transmits the data to its nearest neighbor in given level of hierarchy. Nodes that receive data at lower level rise at the next level. So this paper, they have analyzed the performance of concentric cluster based PEGASIS for WSNs. Simulation of this paper shows that when number of nodes, energy consumption per bit and number of data bits decreases, energy consumption decreases.

\section{B. Survey of Energy-Efficient Hierarchical Cluster-Based Routing in Wireless Sensor Networks}

One of the important issues in wireless sensor network is limited battery power. For this reason battery power is crucial parameter in the algorithm design to increase lifetime of nodes in the network. To maximize the lifetime of sensor nodes, it is preferable to distribute the energy dissipated throughout the wireless sensor network in order to maximize overall network performance. Clustering can be used as an energy-efficient communication protocol. The main idea of clustering is to minimize the total transmission power aggregated over the nodes in the selected path, and to balance the load among the nodes for prolonging the network lifetime. Clustering is a sample of layered protocols in which a network is composed of several clumps (clusters) of sensors. Each clump is managed by a special node or leader, called cluster head $(\mathrm{CH})$, which is responsible for coordinating the data transmission activities of all sensors in its clump which shown in below figure

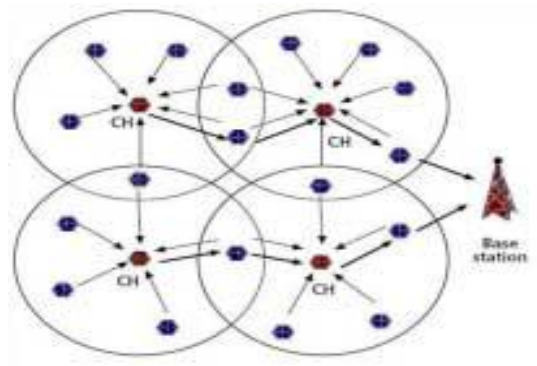

Figure 2 Clustering Of Sensor Network ${ }^{[5]}$

All sensors in a cluster communicate with a cluster head $(\mathrm{CH})$ that acts as a local coordinator or sink for performing intra-transmission arrangement and data aggregation. Cluster heads transmits the sensed data to the global sink. To transmit the data from member node to cluster head is smaller compared to their respective distance to the global sink.

Advantages of hierarchical PEGASIS protocol over conventional PEGASIS protocol

- Distance to transmitting data is reduced in PEGASIS.

- Message overhead is reduced in PEGASIS

- Since each node gets selected once, energy dissipation is balanced among sensor nodes.

Since a network is characterized by its limited wireless channel bandwidth, it would be beneficial if the ratio of data transmitted to the sink can be reduced. To get the advantage, a local collaboration between the sensors in a cluster is required in order to reduce bandwidth demands. Clustering usually localizes the routing setup within the cluster and therefore it reduces the routing overhead. Using clustering, the network appears smaller and more stable. The information, generated from neighboring sensor nodes, is often redundant and highly 
correlated, so data aggregation by each cluster head conserves communication bandwidth as well. Moreover, the ability to use different power levels in inter-cluster and intra cluster communication reduces the interferences and the collisions in the network resulting in a better throughput. Clustering is a challenging task. $\mathrm{CHs}$ often lose more energy compared to regular nodes. It is necessary to perform re-clustering periodically in order to select energy-abundant nodes to serve as CHs. This re-clustering distributes the load uniformly on all the nodes.

\section{GRID-PEGASIS: A Data Gather Scheme with Novel Chain Construction for Wireless Sensor Networks}

The energy efficiency and balancing energy dissipation are important issues for the data gather schemes of WSNs. In this paper, GRID-PEGASIS is proposed to improve the performance of chain-based scheme on these two issues. GRID-PEGASIS divides the sensing area into several small cells to reduce the hop variance of a sensing area chain. With the smallest hop variance, GRID-PEGASIS balances the energy consumption for nodes. Basically, if the spread area of sensors is small, the probability of creating long hops in a chain will be small. So, the idea of the proposed chain construction is to divide the sensing area into several small grid cells as shown in figure.

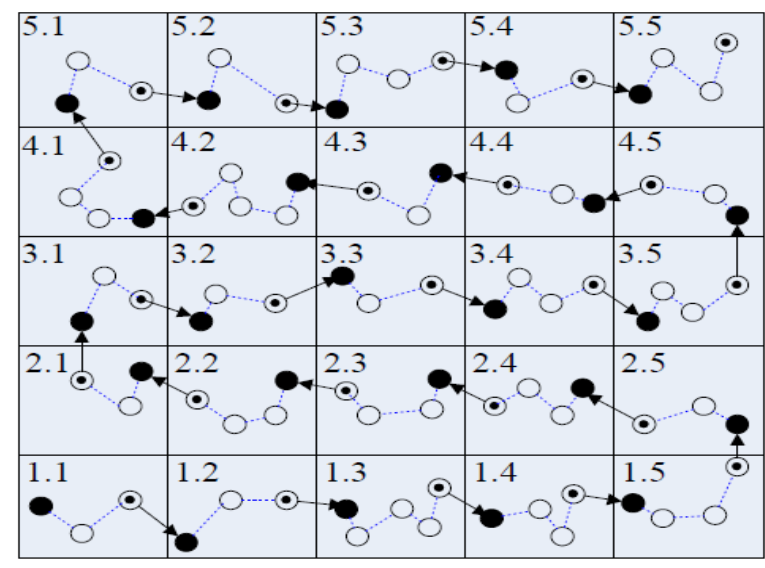

End node

Start node

Sensor node

Figure 3 GRID-PEGASIS ${ }^{[8]}$

For example, the start node of the cell chain in cell 5.2 will connect with the end node of the cell chain in cell 5.1. Nodes in each cell first form a chain (cell chain) by using the Greedy algorithm. Then the start node of each cell chain will connect with the end node of the cell chain in neighbor cell. By cascading all cell chains, all sensors will form a chain (area). After constructing the area chain, the GRID-PEGASIS select an area chain head. Then, the area chain head collects the data from other nodes and sends the data to the BS. Simulation of this paper results show that, With the smallest hop variance, GRID-PEGASIS improves the balance of energy consumption for nodes. GRID-PEGASIS can largely prolong the network lifetimes whose definitions are the times of the first $1 \%, 5 \%$ and $25 \%$ nodes running out of energy.

\section{Problem STATEMENT}

PEGASIS is a near optimal chain-based protocol. The basic idea of the protocol is that in order to extend network lifetime, nodes need only communicate with their closest neighbors and they take turns in communicating with the base-station. When the round of all nodes communicating with the base-station ends, a new round will start and so on. This reduces the power required to transmit data per round as the power draining is spread uniformly over all nodes.

As previously discussed the PEGASIS mainly works in the scenario like nodes have same properties or characteristics that mean the nodes are homogeneous. So it is so easy to maintain this kind of homogeneous environment. But with the help of heterogeneous nodes, energy can be saved. It is somehow complex to maintain that kind of environment.

\section{SOLUTION}

Till now PEGASIS protocol is only for homogeneous environment. So new improvement on PEGASIS: PEGASIS for heterogeneous environment is proposed. 
PEGASIS is based on LEACH protocol. Following assumption has been made

- The heterogeneity of environment is defined on energy level and battery to be used.

- $\quad$ Two type of node for heterogeneity, type $\mathrm{A}$ is for $\mathrm{CH}$ and type $\mathrm{B}$ for its member.

- $\quad$ Solar cell are used as battery support for TypeA nodes while AA battery is used for TypeB

- $\quad$ Nodes are static.

Algorithm:

Parameters used for Protocol:

$\mathrm{P}=$ probility of node participating in cluster-head formation

$\mathrm{N}=$ number of nodes

type $\mathrm{A}=$ nodes containing solar battery

type $\mathrm{B}=$ nodes containing $\mathrm{AA}$

ran $=$ random number

$\mathrm{I}, \mathrm{j}=$ neighbor node

disFromSink= distance From sink;

1. If(typeA) then,

2. ran is generated by random function

3. $\quad$ If $(\operatorname{ran}<\mathrm{p})$

4. Select as Cluster-Head $(\mathrm{CH})$ then,

5. Broadcast advertisement message

6. Other node will join to cluster head is based on RSSI value.

Algorithm for chain construction:

1. If(isCH) then

2. $\quad \operatorname{If}(\operatorname{distFromSink}(\mathrm{i})<\operatorname{disFromSink}(\mathrm{j}))$ then

3. $\quad$ Send join message to cluster-Head i

4. Else

5. Send join message to cluster-head $\mathrm{j}$

\section{CONCLUSION}

PEGASIS is one of the protocol which is energy efficient and increases the network lifetime. It is based on chain construction and data gathering approach. All the implementation of the PEGASIS protocol till now is only for the homogeneous nodes. In future, heterogeneous environment will develop for better result.

\section{ACKNOWLEDGMENT}

I would like to thanks who help me to do so. And finally a special thanks goes to my internal guide Prof. C.R. Parekh, HOD-EC, GEC Gandhinagar, who help me to assemble the parts and gave suggestion about my interest area.

\section{REFERENCES}

[1] Evolution-of-wireless-sensor-networks, Silicon Labs

[2] An Introduction to Wireless Sensor Networks, Bhaskar Krishnamachari

[3] Wireless Sensor Networks: Motivation, Progress, and Challenges Tracy Camp Colorado School of Mines.

[4] Prof. Dr. Dieter Hogrefe Dr. Omar Alfandi, wireless ad hoc and sensor network

[5] Shio Kumar Singh,M P Singh,D K Singh," A Survey of Energy-Efficient Hierarchical Cluster-Based Routing in Wireless Sensor Networks"

[6] Stephanie Lindsey and Cauligi S. Raghavendra," PEGASIS: Power-Efficient GAthering in Sensor Information Systems"

[7] Liu Yueyang, Ji Hong, Yue Guangxin "An Energy-Efficient PEGASIS-Based Enhanced Algorithm in Wireless Sensor Networks"

[8] Yung-Fa Huang and Liang-Chun Tai, Jen-Yung Lin" GRID-PEGASIS: A Data Gather Scheme with Novel Chain Construction for Wireless Sensor Networks"

[9] Neha Rathi, Partha Pratim, Kaushik Ghosh "Performance Analysis of Concentric Cluster based PEGASIS for Wireless Sensor Networks"

[10] Meenu, Vandana," Modified PEGASIS in WSN to increase network lifetime" 